\title{
Chains, clumps, and strings: Magnetofossil taphonomy with ferromagnetic resonance spectroscopy
}

\author{
Robert E. Kopp a, ${ }^{\text {, }}$, Benjamin P. Weiss ${ }^{\text {b }}$, Adam C. Maloof ${ }^{\text {b,c }}$, Hojotollah Vali ${ }^{\text {d,e }}$, \\ Cody Z. Nash ${ }^{\text {a }}$, \& Joseph L. Kirschvink ${ }^{\text {a }}$ \\ a. Division of Geological and Planetary Sciences, California Institute of Technology, Pasadena, CA 91125, USA \\ b. Department of Earth, Atmospheric, and Planetary Sciences, Massachusetts Institute of Technology, Cambridge, \\ MA 02139, USA \\ c. Present address: Department of Geosciences, Princeton University, Princeton, NJ 08544, USA \\ d. Department of Anatomy and Cell Biology and Facility for Electron Microscopy Research, McGill University, \\ Montréal, QC, Canada H3A 2B2 \\ e. Department of Earth and Planetary Sciences, McGill University, Montréal, QC, Canada H3A 2A7 \\ * Corresponding Author: Division of Geological and Planetary Sciences, MC 170-25, California Institute of \\ Technology, Pasadena, CA 91125, USA. Tel.: +1-626-395-2949; fax: +1-626-568-0935. \\ E-mail addresses: rkopp@caltech.edu (R. E. Kopp), bpweiss@mit.edu (B. P. Weiss), maloofa@mit.edu (A. C. \\ Maloof),vali@eps.mcgill.ca (H. Vali), cody@caltech.edu (C. Z. Nash), kirschvink@caltech.edu (J. L. Kirschvink)
}

Received 15 February 2006; received in revised form 26 April 2006; accepted 1 May 2006 Editor: S. King

\begin{abstract}
Magnetotactic bacteria produce intracellular crystals of magnetite or greigite, the properties of which have been shaped by evolution to maximize the magnetic moment per atom of iron. Intracellular bacterial magnetite therefore possesses traits amenable to detection by physical techniques: typically, narrow size and shape distributions, single-domain size and arrangement in linear chains, and often crystal elongation. Past strategies for searching for bacterial magnetofossils using physical techniques have focused on identifying samples containing significant amounts of single domain magnetite or with narrow coercivity distributions. Searching for additional of traits would, however, increase the likelihood that candidate magnetofossils are truly of biological origin. Ferromagnetic resonance spectroscopy (FMR) is in theory capable of detecting the distinctive magnetic anisotropy produced by chain arrangement and crystal elongation. Here we present analyses of intact and lysed magnetotactic bacteria, dilutions of synthetic magnetite, and sedimentary samples of modern carbonates from the Great Bahama Bank, Oligocene-Miocene deep-sea muds from the South Atlantic, and Pleistocene lacustrine deposits from Mono Basin, California. We demonstrate that FMR can distinguish between intact bacterial magnetite chains, collapsed chains, and linear strings of magnetite formed by physical processes. We also show that sediments in which the magnetization is likely carried by bacterial magnetite have FMR spectra resembling those of intact or altered bacterial magnetite chains.
\end{abstract}

Keywords: magnetotactic bacteria; biogenic magnetite; ferromagnetic resonance; magnetofossils 


\section{Introduction}

Magnetotactic bacteria are a polyphyletic group of organisms that, uniquely among the bacteria, engage in biologically organized mineralization. They precipitate intracellular crystals of ferrimagnetic iron minerals, either magnetite $\left(\mathrm{Fe}_{3} \mathrm{O}_{4}\right)$ or greigite $\left(\mathrm{Fe}_{3} \mathrm{~S}_{4}\right)$, within membranebound organelles called magnetosomes. Although magnetosomes likely have multiple functions [1,2], one major role is the passive alignment of bacterial cells with the geomagnetic field. Magnetotactic bacteria tend to live in regions with well-defined redox gradients. Everywhere except on Earth's geomagnetic equator, the geomagnetic field has a vertical component; by swimming along the field lines, the bacteria reduce a threedimensional search for optimal chemical conditions to a one-dimensional search [3-5].

Because natural selection can lead magnetotactic lineages to maximize their magnetic sensitivity for the amount of iron used, magnetite crystals produced by magnetotactic bacteria have a number of distinctive traits that facilitate their identification as magnetofossils in sedimentary deposits [6]. Not all magnetotactic organisms produce magnetite with all of these traits, and abiotic processes can produce crystals with some of them, but the greater the number of traits present, the higher the degree of confidence with which one can identify magnetite as biogenic. The following traits have been identified so far. Magnetite from magnetotactic bacteria is generally chemically pure and lacking in crystallographic defects (but see the relatively rare anomalies described by [7]). It almost always has a narrow size and shape distribution, is stably or meta-stably single domain, and is arranged in chains of particles. The crystals also tend to have unusual crystal morphologies that increase the single domain stability field $[8,9]$ : sometimes elongated along the magnetocrystalline easy axis, and often with truncated crystal edges.

Because magnetotactic bacteria prefer specific chemical environments, their fossils convey information about paleoclimate and paleoecology $[10,11]$. Magnetofossils also have a major advantage over other bacterial fossils: because the fossils are magnetic, samples likely to contain magnetofossils can potentially be identified using physical techniques rather than time-consuming electron microscopy surveys. Nevertheless, the pre-Quaternary fossil record of magnetotactic bacteria is sparse. The oldest samples with chains of single domain particles with distinctively biological morphologies are Cretaceous chalks from England [12], although a few putative Precambrian magnetofossils have also been identified $[4,13]$.

Previous attempts to search for magnetofossils in pre-Quaternary sediments with physical techniques have relied on the use of basic rock magnetic procedures designed to identify single domain magnetite [e.g. 13]. However, single domain magnetite may be precipitated or concentrated by abiotic processes and is not unique to magnetofossils. Finding additional characteristic traits would greatly strengthen identifications. New techniques for unmixing coercivity spectra $[14,15]$ allow determination of the degree of variation within populations of magnetic particles, and thus allow detection of the narrow particle size and shape distributions characteristic of magnetotactic bacteria and magnetofossils. Acquiring high-resolution coercivity spectra is a time-intensive process, however. Although the process can be abbreviated for examining stratigraphic variation within a section where the endmember components have been identified [11], its time requirements may limit its utility in surveying the deep-time magnetofossil record. 
The magnetofossil chain structure and magnetosome crystal elongation should exert distinctive influences on microscale magnetic anisotropy and should therefore be detectable using physical techniques. For instance, Moskowitz et al. [16] proposed that the relative magnitude of demagnetization upon warming across magnetite's Verwey transition under field cooled and zero-field cooled conditions is an indicator of chain structure. While their test did successfully distinguish fresh bacterial magnetite from other sources of magnetite, it is highly susceptible to crystal oxidation and thus to false negatives. In application to sediments, it also needs to be corrected for mixing of materials [15].

Isolated, equidimensional magnetite particles are dominated by negative cubic magnetocrystalline anisotropy, such that particle energy is highest when magnetization is aligned along the principal axes of the cubic crystal system and lowest when magnetization is aligned with the family of [111] crystal axes. Positive uniaxial shape anisotropy related to crystal elongation and particle organization in linear chains, in contrast, minimizes magnetostatic energy when the magnetization is aligned parallel to the crystal or chain elongation axis and maximizes magnetostatic energy when the magnetization is orthogonal to the crystal or chain elongation axis. Lowering the magnetostatic energy is equivalent to applying a field along the direction of magnetization, while increasing the energy of a state is equivalent to applying a field against the magnetization. Thus, a physical technique capable of assessing the effective field felt by a particle should be sensitive to chain structure and elongation. Ferromagnetic resonance spectroscopy (FMR) is such a technique [17-20].

FMR is a form of electron spin resonance, also known as electron paramagnetic resonance (EPR) [21]. FMR is based on the Zeeman effect, which causes the energy of an electron with spin aligned with a magnetic field to be lower than that of an electron with spin aligned against the field. The energy splitting is given by $\Delta E=g \beta B$, where $g$ is the spectroscopic g-factor, $\beta$ is the Bohr magneton $\left(9.37 \times 10^{-24} \mathrm{Am}^{2}\right)$, and $B$ is the field felt by the electron. The spectroscopic $g$-factor is 2.0023 for a free electron and 2.12 for an electron in magnetite $[21,22]$. The electron can therefore absorb a photon with energy $h v=g \beta B$.

In a conventional EPR spectrometer, a sample is placed in a resonating cavity situated inside an electromagnet. A microwave radiation source with limited frequency adjustability (typically X-band, 9.0-9.8 GHz) generates photons. A detector measures the first derivative of absorption as the electromagnet sweeps the magnetic field strength across a range of values [21]. For a single crystal, the resonant field is shifted to lower values when magnetization is aligned along a magnetically easy direction (e.g., along the [111] crystal axis of magnetite, or along the elongation axis of an elongate crystal or chain) and to higher values when the magnetization is aligned along a hard direction.

Most samples of geological interest are not single crystals, but are composed of particles in a variety of arrangements and orientations with respect to the applied magnetic field. The spectrum of a powder of magnetostatically non-interacting, spherical (isotropic) particles is simply a broadened form of the single crystal spectrum (Fig. 1a), but for powders of anisotropic particles, the spectrum not only broadens but also develops asymmetry (Fig. 1b-e). Consider the case of a powder of elongate magnetite crystals, with elongation axes distributed uniformly in all directions (Fig. 1c). From simple geometrical considerations, there will be a small number of particles with elongation axes closely aligned with the direction of the applied field, and many more with elongation axes aligned close to the plane perpendicular to the applied field. Those with elongation axes aligned with 
the applied field will absorb at lower fields than would isotropic particles, while those with elongation axes in the normal plane will absorb at higher fields. Thus, compared to the spectrum of a powder of isotropic particles, the peak absorption will be shifted to higher field values (i.e., lower effective $g$-factor) but will have a tail asymmetrically extended in the low field direction. Conversely, a powder of particles with negative anisotropy will have increased effective $g$-factors and high field extended asymmetry (Fig. 1b and 1d).

As can be seen in Figs. 1c and 1d, the derivative spectrum of a powder of particles with uniaxial anisotropy has two peaks on the asymmetrically extended side. The one closer to the zero crossing is associated with the rise to the peak of absorption, while the one farther from the zero crossing is associated with the transition from zero absorption to positive absorption. Samples with more complex anisotropies may have additional peaks. These differences, however, can be obscured by factors that symmetrically broaden linewidth, such as magnetostatic interactions and sample heterogeneity (Fig. 1e).

Weiss et al. [20] found that bacterial magnetite chains have FMR spectra distinct from abiotic magnetite. They postulated that the unique spectra of magnetosome chains result from the positive magnetic anisotropy of the chain structure (Fig. 1c), which contrasts with the negative cubic magnetocrystalline anisotropy of roomtemperature magnetite (Fig. 1b). They demonstrated, through measurements of laboratory cultures, that intact magnetotactic bacteria have spectra with low-field extended asymmetry, as well as effective $g$-factors shifted below the standard magnetite value of 2.12 and two local maxima. Weiss et al. [20] also showed that lysed (broken) chains do not have the distinct FMR signature of intact chains, which suggests that the chain structure plays a major role in the FMR signature. However, because the crystals in the lysed chains in their experiments were clumped and highly magnetostatically interacting, Weiss et al. were unable to measure the FMR spectra of isolated magnetosomes and thus to demonstrate that the characteristic FMR spectra of magnetosome chains arises from the chain structure rather than from some trait of the individual crystals.

In this paper, we investigate the effects of chain structure and crystal elongation on FMR spectra and the extent to which these features can be mimicked by process other than the growth of chains of magnetite within magnetotactic bacteria. We examined intact and disrupted chains of magnetite from Magnetospirillum magneticum strain AMB-1, as well as dilutions of magnetite from lysed AMB-1 and of abiogenic magnetite strung out into linear features. We also examined sediments from a modern carbonate platform in the Bahamas, Oligocene-Miocene clays and calcareous oozes from the Atlantic Ocean, and lacustrine silts and muds from Mono Basin, California, in an attempt to determine whether these features corresponded to those observed in nature.

\section{Samples}

\subsection{Bacterial cultures}

For the lysis experiments, $2 \mathrm{~L}$ of $M$. magneticum strain AMB-1 (ATCC strain 700264) were grown to early stationary phase, at $\sim 10^{8}$ cells $/ \mathrm{mL}$, using standard culture conditions [23] and then spun down into three subsamples, which were resuspended in $5 \mathrm{~mL}$ Tris buffer. Five $\mu \mathrm{L}$ of $\beta$-mercaptoethanol were added to subsample A3. The samples were frozen at $-20^{\circ} \mathrm{C}$ and then thawed at $5^{\circ} \mathrm{C}$. Then, $270 \mathrm{mg}$ of sodium dodecyl sulfate (SDS) were added as a detergent to A3, which was incubated at room temperature for ten minutes. A2 and A3 were subjected to ultrasonication with a Fischer Scientific Sonic Dismembrator 550 for six minutes, with pulses of 0.5 seconds alternated with pauses of 0.5 seconds. 
Next, the samples were spun down, frozen, and freeze-dried. Half of A3 was diluted to $\sim 0.1 \%$ in powdered sucrose by grinding in an agate mortar and pestle, first for one minute (to form subsample A3b) and then for five minutes (to form subsample A3c). Halves of subsamples A1 and A2 were similarly diluted by grinding for five minutes to form subsamples $\mathrm{A} 1 \mathrm{~b}$ and $\mathrm{A} 2 \mathrm{~b}$.

Based on rock magnetic data and transmission electron microscope images acquired in previous studies from the related magnetotactic bacterium M. magnetotacticum strain MS-1 [24, 25], we expect that ultrasonicated AMB-1 contains chains of magnetite crystals liberated from cells but remaining bound by magnetosome membranes. The crystals are likely strung together in composite chains formed by linkage of individual chains from different cells, with some of the chains closed to form loops (see Fig. 1 of [25]). Closed loops represent the minimum energy configuration for four or more equidimensional magnetite crystals [26].

SDS treatment, in contrast, destroys both cytoplasmic and magnetosome membranes. As a consequence, the crystals in SDS-treated samples collapse into agglomerates with strong three-dimensional magnetostatic interactions. We predict that, when progressively dispersed to lower density, the crystals minimize their energy by transitioning to increasingly linear "stringy" arrangements, with magnetic dipoles oriented in a head-to-tail fashion [26].

\subsection{Synthetic magnetite dilutions}

TMB-100 is a synthetic magnetite powder produced by the Toda Industrial Company. The powder contains predominantly equidimensional, octahedral crystals of 80-200 nm diameter. In concentrated form, the crystals form magnetostatically interacting masses with diameters of several microns [20].
Aliquots of TMB-100 were mixed in a tube with powdered sucrose. Each mixture was shaken to disperse the magnetite through the sucrose. Undispersed accumulations of magnetite were removed with a small $\mathrm{NdFeB}$ magnet. The final mass ratios of magnetite to sucrose were approximately $6 \%, 6000 \mathrm{ppm}$, and $400 \mathrm{ppm}$ (for samples T1, T2, and T3 respectively). Subsamples of each mixture were powdered with an agate mortar and pestle for one minute and then for four minutes.

\subsection{Sedimentary samples}

Holocene-Recent peritidal carbonate mud samples were collected from a $210 \mathrm{~cm}$ core (C51: N 25'1.35' $\mathrm{W} 78^{\circ} 9.9^{\prime}$ in the WGS84 datum) through a levee crest in the Triple Goose Creek region of Andros Island, the Bahamas. The core was extracted using a Livingstone piston corer provided to us by the Limnological Research Center, University of Minnesota. The core was separated into halves and sediment was collected in nonmagnetic plastic boxes from interior sediment that had not been in contact with any metal objects. The upper $3 \mathrm{~cm}$ of the core is characterized by active microbial mats, containing the morphologically-identified filamentous cyanobacteria Schizothrix [27]. The active microbial mat is underlain by 29 $\mathrm{cm}$ of tan, faintly-laminated mud, which is in turn underlain by $178 \mathrm{~cm}$ of grey, bioturbated carbonate mud, with variable concentrations of $<1 \mathrm{~cm}$ long cerithid gastropod shells.

Samples of Oligocene-Miocene deepsea clay and calcareous nanofossil ooze from the southern Atlantic Ocean were taken from the Deep Sea Drilling Project site 522, cores 12-20 (44-80 mbsf) [28]. The magnetic mineralogy of these samples, which is dominated by biogenic magnetic, was described by Vali and Kirschvink [29]. Samples were provided by the Ocean Drilling Program (ODP), which is sponsored by the U. S. National Science Foundation (NSF) and 
participating countries under management of Joint Oceanographic Institutions (JOI), Inc. Samples of the Pleistocene Wilson Creek Formation of Mono Basin, California [30], were collected with permission of the Forest Service in May 2005 at the formation's type section northwest of Mono Lake (N $38^{\circ}$ $1.3^{\prime} \mathrm{W} 119^{\circ} 7.5^{\prime}$ in the WGS 84 datum). Aside from a few ash layers and sand lenses, the sediments of the Wilson Creek Formation are dominantly laminated (unbioturbated) muds and silts derived from the crystalline rocks of the Sierra Nevada to the west.

\section{Methods}

\subsection{Rock magnetic measurements}

Room temperature magnetic remanence experiments were performed using a 2G Enterprises SQUID magnetometer housed in a magnetically-shielded room.. Anhysteretic remanent magnetization (ARM) was acquired in a $100 \mathrm{mT}$ alternating field and a DC biasing field progressively varied in steps from 0 to $1 \mathrm{mT}$ and was then removed by progressive alternating field demagnetization (AF) up to $160 \mathrm{mT}$. Isothermal remanent magnetization (IRM) at $100 \mathrm{mT}$ was then acquired and removed by $\mathrm{AF}$.

The ARM susceptibility of noninteracting single domain particles is a function of temperature, particle moment, and particle switching field [31]. Threedimensional interparticle magnetostatic interactions significantly reduce ARM susceptibility [32], so comparison of the ARM susceptibility of similar particles in different arrangements reflects changes in particle arrangement. We report ARM susceptibility as $k_{A R M} / I R M$, the ARM acquired per $\mathrm{A} / \mathrm{m}^{2}$ with a biasing field of $0.1 \mathrm{mT}(79.6 \mathrm{~A} / \mathrm{m})$ and an alternating field of $100 \mathrm{mT}$, normalized to the IRM acquired in a field of $100 \mathrm{mT}$. Typical freeze-dried magnetotactic bacteria (weakly interacting) have $k_{A R M} / I R M=2.5$ $\mathrm{mm} / \mathrm{A}$, while a typical chiton tooth (strongly interacting) has $k_{A R M} / I R M=0.09 \mathrm{~mm} / \mathrm{A}$.

Low-temperature experiments were performed using a Quantum Design Magnetic Properties Measurement System in the Molecular Materials Resource Center of the Beckman Institute at Caltech. Field cooled and zero-field cooled curves were acquired by cooling the sample either in a $3 \mathrm{~T}$ field or in zero field to $5 \mathrm{~K}$, respectively, followed by pulsing with a $3 \mathrm{~T}$ field and then measuring the remanence magnetization during warming to room temperature in zero field. The combination of field cooled and zero field cooled measurements constitute the Moskowitz test $[16,20]$. The sample was then cycled through low-temperature by pulsing it with a $3 \mathrm{~T}$ field at room temperature and then measuring the remanent magnetization as the sample was cooled to $10 \mathrm{~K}$ and then warmed to room temperature.

The results of the low-temperature experiments are reported as the parameters $\delta_{Z F C}, \delta_{F C}$, and $f_{L T C}$. The parameters $\delta=\left(J_{80 \mathrm{~K}}-\right.$ $\left.J_{150 \mathrm{~K}}\right) / J_{80 \mathrm{~K}}$ assessed for the zero-field cooled and field cooled curves respectively, where $J_{80 \mathrm{~K}}$ and $J_{150 \mathrm{~K}}$ are the moments measured at 80 $\mathrm{K}$ and $150 \mathrm{~K}$, respectively. A ratio $\delta_{F C} / \delta_{Z F C}>$ 2.0 passes the Moskowitz test and is considered to be an indicator of the presence of magnetosome chains, although partial oxidation and mixing can cause intact chains to fail the test $[16,20]$. Magnetization retained through low-temperature cycling is expressed as the memory parameter $f_{\text {LTC }}=J_{\text {LTC }} / J_{0}$, where $J_{0}$ and $J_{\text {LTC }}$ are respectively the roomtemperature magnetization measured before and after cycling the samples to $10 \mathrm{~K}$.

\subsection{Ferromagnetic resonance spectroscopy}

Ferromagnetic resonance spectra were acquired using an X-band Bruker ESP 300E EPR Spectrometer housed at Caltech. Except for particularly strong samples, microwave power was set at $640 \mu \mathrm{W}$ and spectra were integrated over three sweeps of the applied 
field from 0 to $600 \mathrm{mT}$. For the strongest samples, microwave power was set at $64 \mu \mathrm{W}$ and one sweep was performed. The spectra were measured at $\sim 9.8 \mathrm{GHz}$. For $77 \mathrm{~K}$ measurements, samples were loaded in a quartz glass dewar filled with liquid nitrogen and spectra were measured at $\sim 9.4 \mathrm{GHz}$.

FMR spectra are rich in detail; to extract all the information they convey, they must be examined individually. As a first order summary of spectral characteristics, we use three parameters [20]: $g_{\text {eff }}, A$, and $\Delta B_{\text {FWHM }}$ (Fig. 2). The effective $g$-factor, $g_{e f f}$, is the $g$ factor associated with maximum absorption

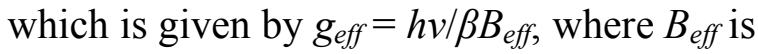
the field value of maximum absorption. The asymmetry ratio is defined as $A=$ $\Delta B_{\text {high }} / \Delta B_{\text {low }}$, where $\Delta B_{\text {high }}=B_{\text {high }}-B_{\text {eff, }} \Delta B_{\text {low }}$ $=B_{\text {eff }}-B_{\text {low }}$, and $B_{\text {high }}$ and $B_{\text {low }}$ are the fields of half maximum absorption at low-field and high-field sides of the absorption peak, respectively. The full width at half maximum, $\triangle B_{F W H M}$, is defined as $\triangle B_{F W H M}=B_{\text {high }}+B_{\text {low }}$. Although all these parameters are derived from the integrated absorption spectrum, FMR spectra are generally displayed as derivative spectra, which reveal much greater detail upon inspection. The empirical parameter $\alpha$, generated by linear discriminant analysis [33] to maximize the difference between magnetosome chains and detrital magnetite in $A$ vs. $\triangle B_{F W H M}$ space and discussed at length later, is defined as $\alpha=$ $0.17 A+9.8 \times 10^{-4} \Delta B_{F W H M} / \mathrm{mT}$.

\subsection{Simulation of ferromagnetic resonance spectra}

We used MATLAB code, written following Griscom [34, 35], to simulate FMR spectra and thereby allow us to understand the relationship between the empirical parameters and physical parameters. We assume that the simulated material is a powder of dilute single-domain particles, small with respect to the microwave skin depth $(\sim 5 \mu \mathrm{m}$ in magnetite [34]), with the particles uniformly distributed in all orientations, and that, at resonance, the orientation of the moment vector approximates the field orientation. Magnetostatic interactions were neglected except as a source of anisotropy, and all anisotropy, whether arising from magnetocrystalline anisotropy, shape anisotropy, or particle arrangement, was treated through a single pair of anisotropy constants, $K_{1}$ and $K_{2}$.

The orientation of an individual particle is defined by the angles $\theta$ and $\varphi$, where $\theta$ is the angle between the applied DC field and the axis of the particle and $\varphi$ is the angle of the axis out of the plane defined by the microwave field and the applied DC field. For a particle with uniaxial anisotropy, the first-order resonance condition (derived from the anisotropy energy $E=K_{1} \sin ^{2} \theta+K_{2} \sin ^{4}$ $\theta$ following the approaches of $[18,35])$ is given by $B_{r e s i n c}(\theta)=B_{\text {mere }}-\frac{1}{2} B_{m m} \times\left[3 \cos ^{2} \theta-1+\left(K_{2} / K\right)\left(8 \cos ^{2} \theta \sin ^{2} \theta-2 \sin ^{4} \theta\right)\right]$ where $B_{\text {true }}=h v / \beta g_{\text {true }}$ is the resonance field in the absence of anisotropy, $B_{\text {an }}$ is the anisotropy field $2 K_{1} / M_{\mathrm{s}}, K_{1}$ and $K_{2}$ are the first-order and second-order uniaxial anisotropy constants, and $M_{s}$ is the saturation magnetization. For cubic anisotropy, the resonance condition of [35] was used.

To compute the powder absorption at applied field $B_{a p p}$, a Gaussian broadening function of linewidth $\sigma$ is applied and spectra are numerically integrated over all solid angles:

$$
A\left(B_{a p p}\right)=\int_{\theta=0}^{\pi / 2} \int_{\phi=0}^{2 \pi} \frac{\exp \left(-\left(B_{a p p}-B_{\text {res }}(\theta, \phi)\right)^{2} / 2 \sigma^{2}\right)}{\sqrt{2 \pi} \sigma} d \phi \sin \theta d \theta
$$

Several physical effects are subsumed in the Gaussian broadening; in natural samples, a major cause of broadening is heterogeneity in particle size, shape, and arrangement [36].

While not capable of fitting our observed spectra perfectly, nonlinear least square fitting using these model spectra provides reasonably good approximations. For almost all our samples, second-order uniaxial fits were better than second-order cubic fits, 
and they never were significantly worse. We would expect this to be the case for samples in which magnetostatic interactions or shape anisotropy dominated the internal magnetic environment.

\subsection{Transmission electron microscopy (TEM)}

We prepared replicas, composed of a 1-2 nm thick metal thin film of $95 \%$ platinum and $5 \%$ carbon and a supporting carbon film $15-20 \mathrm{~nm}$ thick, of the synthetic magnetite dilutions to allow us to remove the sucrose dilutant while preserving the relationship between the dilutant and the magnetite. Ten $\mu \mathrm{L}$ of $\sim 20 \mathrm{mg}$ of powder dispersed in $99 \%$ pure methanol were transferred onto a freshly cleaved mica thin plate. As the powder settled, the fluid was removed by blotting with filter paper. The replica was prepared in a freeze-fracture unit (Baltec 60, Lichtenstein) at room temperature under high vacuum $\left(10^{-6}\right.$ Torr), as described in [37], by depositing a fine granular Pt-C film from an evaporating source at an incident angle of $30^{\circ}$ and a distance of $15 \mathrm{~cm}$ to the mica plate. Variable densities of Pt-C accumulate along the topographic slopes of the specimen that are facing, oblique to, or shielded from the source, emphasizing the topographic contrast for TEM imaging. To strengthen the ultrathin Pt films and preserve their integrity during cleaning steps, a supporting carbon film was immediately condensed on the $\mathrm{Pt}$ film by a vertical electron beam gun sputtering a carbon rod perpendicular to and $12 \mathrm{~cm}$ away from the surface of the specimen. The replicas were cleaned with distilled water to remove the sucrose and transferred onto 200 mesh formvar-coated $\mathrm{Cu}$ grids.

Imaging was carried out in bright-field illumination with a JEOL JEM-2000FX transmission electron microscope, at an accelerating voltage of $80 \mathrm{kV}$ and magnifications from 5000x-50,000x, and a Gatan 792 Bioscan 1k x 1k Wide Angle Multiscan CCD camera.

\section{Results}

\subsection{Lysis of magnetotactic bacteria}

When comparing different arrangements of similar particles, ARM susceptibility quantifies magnetostatic interactions. Three-dimensional magnetostatic interactions shield particles from the DC biasing field, which causes materials with greater interactions to exhibit lower ARM susceptibility. (This is not the case for materials with one-dimensional magnetostatic interactions, as in magnetosome chains.) As expected from these considerations, the ARM susceptibilities of the samples are ordered: intact AMB-1 (samples A1a and A1b) > ultrasonically disrupted AMB-1 (samples A2a and samples A2b) $>$ dispersed, SDS-treated AMB-1 (sample A3c) > concentrated, SDStreated AMB-1 (samples A3b and A3a) (Fig. 3a, Table 1).

Ferromagnetic resonance spectra of the disrupted AMB-1 samples reflect a shift from linear chains to highly interacting clumps (Fig. 3b, Table 1). The ultrasonically disrupted AMB-1 (sample A2a) exhibits little change in spectrum shape or in the spectral parameters compared to intact AMB-1 (sample A1a). This suggests that, although the magnetostatic interactions as revealed by the ARM curves have increased relative to the intact cells, the local magnetic environment felt by each individual crystal remains dominated by head-to-tail axial interactions; the chains are still intact, although they are no longer separated from one another by cell material (see Fig. 1 of [25]). In contrast, the spectrum of the SDS-treated cells exhibits a high-field extended asymmetry and high $g_{\text {eff }}$ (sample A3a), consistent with the observations of Weiss et al. [20].

The diluted SDS-treated cells (sample A3c) exhibit a spectrum that more closely resembles that of the intact and ultrasonicated samples, although the spectrum of $\mathrm{A} 3 \mathrm{c}$ is a bit 
wider. One key trait of this spectrum that is not readily apparent from the parameterization is its degree of sharpness. The derivative spectra of intact and ultrasonicated AMB-1 (A1a and A2a) have two local maxima, which indicate a low degree of peak broadening caused by heterogeneous particle arrangements. Each particle experiences roughly the same magnetic anisotropy produced by particle interactions within the chain, because the magnetite chains are produced under biological control. In contrast, the dispersed, SDS-treated AMB-1 (A3c) has only one local maximum, likely because the particles are arranged in one-dimensional strings that form through physical processes and therefore have more heterogeneous magnetic anisotropy. The same effect is seen in comparing Fig. 1c and Fig. 1e.

All the AMB-1 spectra are fairly well fit by second-order uniaxial model spectra. The intact, ultrasonicated, and dispersed SDStreated samples have $B_{\text {an }}$ values around 80 $\mathrm{mT}$. Following the method of Butler and Banerjee $[38,39]$, this value is equivalent to the shape anisotropy of an elongate magnetite crystal with a 2.2:1 length-to-width ratio.

Since the crystals produced by AMB-1 are equidimensional, the apparent shape anisotropy must be due to linear magnetostatic interactions. The $-81 \mathrm{mT} B_{a n}$ value of the concentrated SDS-treated sample is equivalent to a single crystal with a $1: 1.1$ length-to-width ratio, and may be due to planar magnetostatic interactions, as on the surface of a clump. All SDS-treated samples have larger $\sigma$ values than intact and ultrasonicated samples, which likely reflect greater heterogeneity of particle arrangement.

The FMR spectra measured at $77 \mathrm{~K}$ are consistent with these findings (Fig. 3b, Table 2). Below the Verwey transition, which, as measured in the FC and ZFC curves (not shown; see also [20]), occurs at $\sim 100 \mathrm{~K}$ in magnetite from AMB-1, magnetite shifts from a cubic crystal system to a monoclinic crystal system and experiences a large increase in magnetocrystalline anisotropy, with the anisotropy constants becoming positive [40, 41]. Thus, the spectra broaden considerably, $g_{\text {eff }}$ shifts to higher values, and $A$ becomes lower, reflecting the positive anisotropy. The last effect is strongest in sample A3a, which shifts from high field asymmetry to mild low field asymmetry, presumably as the anisotropy field comes to dominate magnetostatic interactions. A3a continues, however, to have higher $g_{\text {eff, }} A$, and $\Delta B_{\mathrm{FWHM}}$ than the other samples.

Low-temperature data (Fig. 3c; Table 1) exhibit increasing loss of remanence upon warming or cooling through the Verwey transition with increasing magnetostatic interactions (decreasing ARM susceptibility), consistent with [42]. In addition, the $\delta_{\mathrm{FC}} / \delta_{\mathrm{ZFC}}$ ratio decreases with chain breakdown and only the intact AMB-1 cells pass a Moskowitz test with $\delta_{\mathrm{FC}} / \delta_{\mathrm{ZFC}}>2$. Increased chain breakdown also increases the fraction of remanence loss on cooling and re-warming through the Verwey transition. Curiously, the SDS-treated cells, both concentrated and dispersed, experience a slight increase in remanence when they are cooled through the Verwey transition, while the samples with intact chains lose remanence across the Verwey transition.

\subsection{Dilutions of abiogenic magnetite}

To test our hypothesis that the lowfield extended asymmetry of the dispersed, lysed AMB-1 is a product of physical string formation, we performed analogous experiments using synthetic magnetite. As expected, dilution of the powder decreases three-dimensional magnetostatic interactions, which is reflected in increased ARM susceptibility (Fig. 4a, Table 3). This pattern is consistent when comparing different ratios of magnetite to sucrose and when comparing shaken mixtures to mixtures ground with a mortar and pestle for four minutes. The ARM susceptibility of the samples is ordered: 400 
ppm ground mixture (sample T3c) $>0.6 \%$ ground mixture (sample T2c) $>400 \mathrm{ppm}$ shaken mixture (sample T3a) $>6 \%$ ground mixture (sample T1c) $>0.6 \%$ shaken mixture (sample T2a) $>6 \%$ shaken mixture (sample T1a), although the ARM/IRM ratios of T1c cross above those of $\mathrm{T} 3 \mathrm{a}$ and $\mathrm{T} 2 \mathrm{c}$ at higher biasing fields.

The FMR spectra of these samples reveal the same trend, with $g_{\text {eff }}$ and $\triangle B_{F W H M}$ decreasing in the same order that ARM susceptibilities decrease (Fig. 4b, 5 and Table 3 ). The $6 \%$ shaken mixture is markedly different from the other mixtures. It alone exhibits high-field extended asymmetry, rather than low-field extended asymmetry. The other mixtures exhibit low-field extended asymmetry, which we suggest is due to the formation of strings of particles. If the shift to low-field extended asymmetry were due to changes in crystal shape upon powdering, it would not be present in samples diluted only by shaking. The fitted $B_{\text {an }}$ of the particles is equivalent to that of single magnetite crystals with length-to-width ratios of $\sim 3: 1$, even though TEM images show largely equidimensional crystals. TEM images of sample T2c (Fig. 6) indicate that, as predicted, many of the crystals are arranged in strings. While the strings are much more irregular than biogenic chains, they produce the appropriate magnetic anisotropy to generate FMR spectra with low-field extended asymmetry. Their heterogeneity, however, leads to a broader spectrum than those produced by bacterial magnetite chains; the strings of magnetite have only a single lowfield derivative peak, in contrast to the double peak of the bacterial chains.

\subsection{Sedimentary samples}

The sedimentary samples studied come from three sources: the Bahamas, the southern Atlantic Ocean, and Mono Basin, CA. The range of FMR parameters from these samples are shown in Fig. 7 and in Table 4.
Although no samples from Andros Island have been previously studied using FMR, magnetic measurements and electron microscopy on a small number of samples from elsewhere in the Great Bahama Bank suggest that magnetotactic bacteria are common at the sediment/water interface [43], and data from Pliocene-Pleistocene sediments on San Salvador Island and from the Clino core drilled through the Great Bahama Bank indicate that biogenic magnetite can be preserved in sediments that have undergone diagenesis [20, 44, 45].

The spectra of the Bahamian samples all exhibit multiple derivative maxima and low-field extended asymmetry, with $A$ between 0.71 and $0.86, g_{\text {eff }}$ between 2.01 and 2.11, and $\triangle B_{F W H M}$ between 109 and $192 \mathrm{mT}$ (Fig. 8a). Based on the criteria of Weiss et al. [20], these traits are all consistent with the presence of intact magnetosome chains (Fig. 3). Although many of these parameters also characterize the strings of crystals from lysed cells described above (Fig. 4), the secondary absorption peaks of these Bahamian samples resemble that observed for intact magnetosome chains. In addition, all samples have strong paramagnetic resonances from $\mathrm{Fe}^{+3}(g=4.3)$ and $\mathrm{Mn}^{+2}(g=2.0)$. The latter obscure the magnetite signal in the derivative curve, but due to the sharp peaks of the $\mathrm{Mn}^{+2}$ sextet, the $\mathrm{Mn}^{+2}$ signal is readily removed by curve smoothing (Fig. 7a).

A close examination of core C51 reveals changes with depth suggestive of the progressive alteration of magnetosome chains. The first local maximum of the derivative spectrum in the $6 \mathrm{~cm}$ and $14 \mathrm{~cm}$ samples is well defined. In the 22 and $31 \mathrm{~cm}$ samples, it is present but diminished in strength relative to the second local maximum. In the 42 and $52 \mathrm{~cm}$ samples, the first local maximum is not present, and ARM susceptibility is markedly diminished compared to shallower samples (Fig. 8b, 8c). These changes suggest the progressive degradation of magnetosome chains with depth, although they could 
alternatively represent the mixing of two discrete magnetic materials in the upper sediments, and the loss of one of these with depth.

The deep-sea sediments studied contain magnetofossils of a variety of morphologies, some partially dissolved [29]. TEM images of magnetic extracts from these samples often produced interlocking meshes and linked strings more closely resembling those present in our dilutions of abiogenic magnetite than the chains present in intact bacteria. Excluding one outlier, all the DSDP Leg 73 samples have $A$ of $0.72-1.01, g_{\text {eff }}$ of 2.03-2.11, and $\Delta B_{\mathrm{FWHM}}$ of $136-157 \mathrm{mT}$. A typical spectrum is shown in Fig. 9a. It is similar to that of the dispersed, SDS-treated AMB-1 (sample A3c; dashed line in Fig. 9a). Like sample A3c, it lacks the second local maximum characteristic of intact bacterial cultures and the laminated Bahamian sediments. Instead, it is consistent with more heterogeneous sediments where the magnetization is dominated by bacterial magnetite in which many of the chains have been broken up but the particles have largely remained associated in roughly linear lowenergy configurations - precisely what TEM images [29] reveal.

The samples from the Pleistocene Wilson Creek Formation (Fig. 9b) were deposited in Lake Russell, the late Pleistocene predecessor of modern Mono Lake. Microbiological research in Mono Lake has revealed a variety of magnetotactic organisms (C. Z. Nash, unpublished results), but due to the basin's location in the Sierra Nevada, sedimentary flux into the basin and thus sediment magnetization is dominated by detrital material derived from Sierran intrusive bodies. These samples exhibit a broad range of $A$ values (0.73-1.33; all but two compositionally anomalous samples, an ash and sand lens, have $A>=0.96$ ), have $g_{\text {eff }}$ well above those of all other samples considered in this paper (2.44-3.34; all but the two anomalous samples have $g_{\text {eff }} \geq 2.58$ ), and $\triangle B_{F W H M}$ larger than the samples dominated by bacterial magnetite (207-287 mT).

\section{Discussion}

Weiss et al. [20] suggested that the magnetosome chain structure led cultures of magnetotactic bacteria to produce FMR spectra with multiple derivative maxima, $A<$ 1 and $g_{\text {eff }}<2.12$ (the true g-factor for magnetite). Our results support these claims: our bacterial samples with intact chains had multiple maxima, $A \leq 0.84$ and $g_{\text {eff }} \leq 2.02$. With two exceptions, our sedimentary samples in which magnetofossils are believed to be a major carrier of magnetization had $A \leq$ 0.97 and $g_{\text {eff }} \leq 2.11$ (Fig. 6a; Tables 1, 4), although not all had multiple maxima. Yet while $A<1$ and $g_{\text {eff }}<2.12$ may be necessary conditions for a FMR spectrum to be a spectrum of magnetofossils, they are not sufficient. We were able to produce abiogenic samples with $A \leq 0.87$ and $g_{\text {eff }} \leq 2.11$ by dispersing magnetite in a non-magnetic matrix, thus causing the crystals to string out in minimum energy head-to-tail configurations (Table 3). This finding strengthens the claim that $A<1$ and $g_{\text {eff }}<2.12$ reflect linear arrangements of crystals, but indicates that these criteria alone cannot uniquely identify magnetofossils.

Closer examination of the FMR spectra suggests alternative criteria that may better distinguish between biogenic and abiogenic magnetite. Increased $\triangle B_{F W H M}$ in a spectrum can have multiple causes, including increased anisotropy (increased $\left|B_{a n}\right|$ in model spectra) and greater sample heterogeneity (increased $\sigma$ in model spectra). Variability among magnetotactic bacteria is largely accounted for by the former: the magnetotactic bacteria strain MV-1, which produces elongate truncated hexaoctahedral crystals, has higher magnetic anisotropy than MS-1 or AMB-1, which produce chains of cubo-octahedral crystals [5]. The higher anisotropy both increases $\triangle B_{F W H M}$ and 
decreases $A$. Similarly, MS-1 has lower $A$ and higher $\triangle B_{F W H M}$ than AMB-1, possibly due to better formed crystals and fewer gaps in their chains than AMB-1. In contrast, in abiogenic samples, heterogeneity plays a major role in controlling $\triangle B_{F W H M}$, and $\triangle B_{F W H M}$ can vary significantly without corresponding decreases in $A$.

The parameter $\alpha$, defined in section 3.2 , is useful in distinguishing between biogenic and abiogenic samples. All the bacterial samples with intact chains, including magnetotactic strains MV-1, MS-1 and MC-1 measured by Weiss et al. [20], possess $\alpha<$ 0.25 , while almost all the lithogenic samples from the Wilson Creek Formation have $\alpha>$ 0.40 . With only two exceptions, the DSDP and Bahamian samples, as well as the most dispersed SDS-treated AMB-1, have $\alpha$ between 0.25 and 0.30 . The most dispersed synthetic magnetite sample we produced had $\alpha=0.34$, and the trend from the most concentrated to the most dilute falls roughly upon a straight line in $\Delta B_{F W H M}$ vs. $A$ space.

Although $\alpha$ is an empirical parameter, it serves as a proxy for the physically meaningful Gaussian linewidth $\sigma$. In the domain of parameter space occupied by magnetotactic bacteria and magnetofossils, $\alpha$ of synthetic spectra varies almost linearly with $\sigma$, and for most values of $B_{a n}, \alpha$ increases monotonically as $\sigma$ increases (Fig. 10). The intact magnetotactic bacteria have nearly constant $\alpha$ values, which suggests the differences among their spectra are predominantly caused by variations of $B_{a n}$. The $B_{a n}$ and $\sigma$ parameters obtained by fitting the bacterial and synthetic samples (Tables 1 and 2) support this hypothesis; the intact and ultrasonicated AMB-1 have $\sigma \approx 30 \mathrm{mT}$, while SDS-treated AMB- 1 have $\sigma \approx 50 \mathrm{mT}$ and the synthetic magnetite powders have $\sigma \geq 64 \mathrm{mT}$. We propose that $\alpha<0.30$ suggests the presence of magnetofossils, with lower $\alpha$ values reflecting more intact chains.

At the moment, the data are not strong enough to support firm claims about the presence or absence of biogenic magnetite based on ferromagnetic resonance spectra alone, except perhaps in the case of bacteria that have undergone minimal diagenetic alteration. Nonetheless, FMR provides a useful guide in the search for magnetofossils, and in sediments in which biogenic magnetite is known to be a major contributor to magnetization, FMR allows rapid assessment of the degree to which the magnetite chains have been disrupted. Its speed may also make it a useful tool in unmixing the magnetic components of sediments, although further work in this area is needed. Furthermore, as TEM of sedimentary biogenic magnetite requires the extraction of magnetite from the sediments, FMR provides the only known technique for detecting the presence of linear arrangements of magnetite crystals in situ.

\section{Conclusions}

1. Linear arrangements of magnetite crystals generally yield FMR spectra with $g_{\text {eff }}<2.12$ and $A<1$. To date, all intact magnetosome chains and almost all sedimentary samples believed to have a significant magnetofossil component fall within this domain, as do physically generated magnetite strings produced by dilution of lysed bacterial magnetite or synthetic magnetite.

2. Intact, biogenic magnetite chains yield derivative spectra with multiple low-field local maxima because their constituent particles experience fairly uniform magnetic anisotropy. Physically generated magnetite strings, which have more heterogeneous magnetic anisotropy, do not exhibit multiple local maxima.

3. Among the magnetotactic bacteria, greater crystal anisotropy produces both higher $\triangle B_{F W H M}$ and lower $A$. MV-1, which produces chains of elongate truncated hexa-octahedral crystals, and MS-1 and AMB-1, which produce chains of cubo-octahedral crystals, 
have nearly identical values of $\alpha=0.17 A+$ $9.8 \times 10^{-4} \Delta B_{F W H M} / \mathrm{mT}$, which is an empirical proxy for symmetric spectral broadening caused by factors such as sample heterogeneity. All magnetotactic bacteria measured have $\alpha<0.25$, while almost all sedimentary samples believed to contain significant biogenic magnetite have $\alpha$ between 0.25 and 0.30 . We suggest that, in samples containing magnetofossils, increasing $\alpha$ reflects a lower magnetofossil content and/or increased diagenetic alteration.

4. FMR provides a powerful tool for searching for samples likely to contain magnetofossils and for assessing the degree of alteration of magnetofossils in samples known to contain them.

\section{Acknowledgements}

We thank J. Grotzinger for access to the Bahamian samples, J. Ewing for field assistance in the Bahamas, G. Rossman, S. Kim, T. Raub, and two anonymous reviewers for helpful discussion, B. Brunschweig and A. Di Bilio for technical assistance with the MPMS and EPR spectrometer, respectively, and the Beckman Institute for funding the use of the MPMS. REK was supported by a NSF Graduate Research Fellowship and a Moore Foundation Fellowship, BPW by the NASA Mars Fundamental Research and NSF Geophysics Programs, ACM and CZN by the Agouron Institute, HV by the Natural Sciences and Engineering Research Council of Canada, and JLK by NASA Exobiology grant NAG5-10618 and the NASA Astrobiology Institute.

\section{References}

[1] R.E. Kopp, C.Z. Nash, J.L. Kirschvink, J.R. Leadbetter, A possible magnetite/maghemite electrochemical battery in the magnetotactic bacteria, Eos 85 (2004) Abst. GP34A-06.

[2] S.L. Simmons, D.A. Bazylinski, K.J. Edwards, South-Seeking Magnetotactic Bacteria in the Northern Hemisphere, Science 311 (2006) 371-374.

[3] J.L. Kirschvink, South-seeking magnetic bacteria, J. Exp. Biol. 86 (1980) 345-347.

[4] S.B.R. Chang, J.L. Kirschvink, Magnetofossils, the magnetization of sediments, and the evolution of magnetite biomineralization, Ann. Rev. Earth Planet. Sci. 17 (1989) 169-195.

[5] D.A. Bazylinski, B.M. Moskowitz, Microbial biomineralization of magnetic iron minerals: Microbiology, magnetism and environmental significance, Geomicrobiology: Interactions Between Microbes And Minerals, Reviews In Mineralogy 35, 1997, pp. 181-223.

[6] K.L. Thomas-Keprta, D.A. Bazylinski, J.L. Kirschvink, S.J. Clemett, D.S. McKay, S.J. Wentworth, H. Vali, E.K. Gibson, C.S. Romanek, Elongated prismatic magnetite crystals in ALH84001 carbonate globules: Potential Martian magnetofossils, Geochim. Cosmochim. Acta 64 (2000) 4049-4081.

[7] A.P. Taylor, J.C. Barry, R.I. Webb, Structural and morphological anomalies in magnetosomes: possible biogenic origin for magnetite in ALH84001, J. Microscopy 201 (2001) 84-106.

[8] J.L. Kirschvink, A seventh criterion for the identification of bacterial magnetofossils, Eos 82 (2001) S131.

[9] A. Witt, K. Fabian, U. Bleil, Threedimensional micromagnetic calculations for naturally shaped magnetite: Octahedra and magnetosomes, Earth Planet. Sci. Lett. 233 (2005) 311-324.

[10] T. Yamazaki, H. Kawahata, Organic carbon flux controls the morphology of magnetofossils in marine sediments, Geology 26 (1998) 1064-1066.

[11] R. Egli, Characterization of individual rock magnetic components by analysis of remanence curves. 3. Bacterial magnetite and natural processes in lakes, Phys. Chem. Earth 29 (2004) 869-884.

[12] P. Montgomery, E.A. Hailwood, A.S. Gale, J.A. Burnett, The magnetostratigraphy of Coniacian late Campanian chalk sequences in southern England, Earth Planet. Sci. Lett. 156 (1998) 209-224.

[13] S.B.R. Chang, J.F. Stolz, J.L. Kirschvink, S.M. Awramik, Biogenic magnetite in stromatolites. 2. Occurrence In ancient sedimentary environments, Precamb. Res. 43 (1989) 305-315.

[14] R. Egli, Analysis of the field dependence of remanent magnetization curves, J. Geophys. Res. 108 (2003).

[15] R. Egli, Characterization of individual rock magnetic components by analysis of remanence curves, 1 . Unmixing natural sediments, Studia Geophys. Geodaet. 48 (2004) 391-446. 
[16] B.M. Moskowitz, R.B. Frankel, D.A. Bazylinski, Rock magnetic criteria for the detection of biogenic magnetite, Earth Planet. Sci. Lett. 120 (1993) 283-300.

[17] C. Kittel, On the theory of ferromagnetic resonance absorption, Phys. Rev. 73 (1948) 155-161.

[18] E. Schlömann, Ferromagnetic resonance in polycrystal ferrites with large anisotropy: General theory and application to cubic materials with a negative anisotropy constant, J. Phys. Chem. Solids 6 (1958) 257-266.

[19] A.H. Morrish, The Physical Principles of Magnetism, J. Wiley, New York, 1965.

[20] B.P. Weiss, S.S. Kim, J.L. Kirschvink, R.E. Kopp, M. Sankaran, A. Kobayashi, A. Komeili, Ferromagnetic resonance and low temperature magnetic tests for biogenic magnetite, Earth Planet. Sci. Lett. 224 (2004) 73-89.

[21] G. Calas, Electron Paramagnetic Resonance, Rev. Mineral. 18 (1988) 513-571.

[22] L.R. Bickford, Jr., Ferromagnetic resonance absorption in magnetite single crystals, Phys. Rev. 78 (1950) 449-457.

[23] A. Komeili, H. Vali, T.J. Beveridge, D.K. Newman, Magnetosome vesicles are present before magnetite formation, and MamA is required for their activation, Proc. Natl. Acad. Sci. 101 (2004) 3839-3844.

[24] Y.A. Gorby, T.J. Beveridge, R.P. Blakemore, Characterization of the Bacterial Magnetosome Membrane, J. Bacteriol. 170 (1988) 834-841.

[25] A. Kobayashi, J.L. Kirschvink, C.Z. Nash, R.E. Kopp, D.A. Sauer, L.E. Bertani, W.F. Voorhout, T. Taguchi, Experimental observation of magnetosome chain collapse in magnetotactic bacteria: sedimentological, paleomagnetic, and evolutionary implications, Earth Planet. Sci. Lett. 245 (2006) 538-550.

[26] A.P. Philipse, D. Maas, Magnetic colloids from magnetotactic bacteria: Chain formation and colloidal stability, Langmuir 18 (2002) 9977-9984.

[27] L.A. Hardie, Sedimentation on the modern carbonate tidal flats of northwest Andros Island, Bahamas, Johns Hopkins University Press, Baltimore, 1977, 202 pp.

[28] K.J. Hsü, J.L. LaBrecque, M.F. Carman, A.M. Gombos, A. Karpoff, J.A. McKenzie, S.F. Percival, N.P. Petersen, K.A. Pisciotto, R.Z. Poore, E. Schreiber, L. Tauxe, P. Tucker, H.J. Weissert, Initial Reports of the Deep Sea Drilling Project, 73, U.S. Govt. Printing Office, Washington, 1984, 798 pp.
[29] H. Vali, J.L. Kirschvink, Magnetofossil dissolution in a paleomagnetically unstable deep-sea sediment, Nature 339 (1989) 203206.

[30] C.R. Denham, A. Cox, Evidence that the Laschamp polarity event did not occur 13,30030,400 years ago, Earth Planet. Sci. Lett. 13 (1971) 181-190.

[31] R. Egli, W. Lowrie, Anhysteretic remanent magnetization of fine magnetic particles, J. Geophys. Res. 107 (2002).

[32] S. Cisowski, Interacting vs. non-interacting single-domain behavior in natural and synthetic samples, Phys. Earth Planet. Inter. 26 (1981) 56-62.

[33] E.S. Keeping, Introduction to Statistical Inference, Dover, New York, 1995, 451 pp.

[34] D.L. Griscom, Ferromagnetic resonance spectra of lunar fines: some implications of line shape analysis, Geochim. Cosmochim. Acta 38 (1974) 1509-1519.

[35] D.L. Griscom, Ferromagnetic resonance condition and powder pattern analysis for dilute, spherical, single-domain particles of cubic crystal structure, J. Mag. Res. 45 (1981) 81-87.

[36] L.E. Drain, The broadening of magnetic resonance lines due to field inhomogeneities in powdered samples, Proc. Phys. Soc. 80 (1962) 1380-1382.

[37] U. Zeile, Fundamentals of cryo preparation and replica technique, 7th Asia-Pacific Electron Microscopy Conference, Singapore, 2000, pp. 342-357.

[38] R.F. Butler, S.K. Banerjee, Theoretical singledomain grain size range in magnetite and titanomagnetite, J. Geophys. Res. 80 (1975) 4049-4058.

[39] J.C. Diaz Ricci, J.L. Kirschvink, Magnetic domain state and coercivity predictions for biogenic greigite $\left(\mathrm{Fe}_{3} \mathrm{~S}_{4}\right)$ : a comparision of theory with magnetosome observations, J. Geophys. Res. 97 (1992) 17309-17315.

[40] A.R. Muxworthy, E. McClellan, Review of the low-temperature magnetic properties of magnetite from a rock magnetic perspective, Geophys. J. Int. 140 (2000) 101-114.

[41] K. Abe, Y. Miyamoto, S. Chikazumi, Magnetocrystalline anisotropy of lowtemperature phase of magnetite, J. Phys. Soc. Japan 41 (1976) 1894-1902.

[42] J.G. King, W. Williams, Low-temperature magnetic properties of magnetite, J. Geophys. Res. 105 (2000) 16427-16436.

[43] D.F. McNeill, Biogenic magnetite from surface Holocene carbonate sediments, Great 
Bahama Bank, J. Geophys. Res. 95 (1990) 4363-4371.

[44] D.F. McNeill, R.N. Ginsburg, S.B.R. Chang, J.L. Kirschvink, Magnetostratigraphic Dating Of Shallow-Water Carbonates From SanSalvador, Bahamas, Geology 16 (1988) 8-12.
[45] D.F. McNeill, J.L. Kirschvink, Early Dolomitization Of Platform Carbonates And The Preservation Of Magnetic Polarity, J. Geophys. Res. 98 (1993) 7977-7986. 


\section{Tables}

Table 1

Summary of FMR and magnetic parameters of bacterial samples

\begin{tabular}{|c|c|c|c|c|c|c|c|c|c|c|c|c|c|}
\hline \multicolumn{2}{|c|}{ Sample } & \multicolumn{4}{|c|}{ FMR Spectrum } & \multicolumn{4}{|c|}{ FMR Uniaxial Fit } & \multicolumn{4}{|c|}{ Magnetic properties } \\
\hline ID & Treatment & $g_{\text {eff }}$ & $A$ & $\begin{array}{c}\Delta B \\
(\mathrm{mT})\end{array}$ & $\alpha$ & $g_{\text {fit }}$ & $\begin{array}{c}B_{\text {an }} \\
(\mathrm{mT})\end{array}$ & $\begin{array}{c}K_{2} / \\
K_{1}\end{array}$ & $\begin{array}{c}\sigma \\
(\mathrm{mT})\end{array}$ & $\begin{array}{c}k_{\mathrm{ARM}} / \\
\text { IRM } \\
(\mathrm{mm} / \mathrm{A})\end{array}$ & $\delta_{Z F C}$ & $\begin{array}{c}\delta_{F C} / \\
\delta_{Z F C}\end{array}$ & $f_{L T C}$ \\
\hline A1a & intact & 2.01 & 0.79 & 96 & 0.23 & 2.08 & 81 & -0.13 & 27 & 2.48 & 0.14 & 2.44 & 0.98 \\
\hline A1b & intact, pwd. & 2.01 & 0.83 & 93 & 0.23 & 2.08 & 86 & -0.20 & 29 & 2.36 & & & \\
\hline $\mathrm{A} 2 \mathrm{a}$ & ultra. & 2.01 & 0.73 & 112 & 0.23 & 2.10 & 84 & -0.06 & 30 & 1.34 & 0.16 & 1.94 & 0.89 \\
\hline $\mathrm{A} 2 \mathrm{~b}$ & ultra., pwd. & 2.02 & 0.78 & 103 & 0.23 & 2.09 & 85 & -0.14 & 31 & 1.31 & & & \\
\hline A3a & SDS, conc. & 2.25 & 1.13 & 208 & 0.40 & 2.20 & -81 & 0.43 & 48 & 0.36 & 0.37 & 1.21 & 0.83 \\
\hline$A 3 b$ & SDS, pwd. $1 \mathrm{~m}$ & 2.14 & 0.89 & 188 & 0.34 & 2.17 & -60 & 0.65 & 48 & 0.36 & & & \\
\hline $\mathrm{A} 3 \mathrm{c}$ & SDS, pwd. $5 \mathrm{~m}$ & 2.07 & 0.76 & 148 & 0.27 & 2.14 & 79 & 0.01 & 50 & 0.66 & 0.26 & 1.39 & 0.86 \\
\hline
\end{tabular}

Table 2

Summary of 77 K FMR parameters

of bacterial and synthetic magnetite samples

\begin{tabular}{c|rccc|crrrr}
\hline & \multicolumn{5}{|c|}{ FMR Spectrum } & \multicolumn{5}{|c}{ FMR Uniaxial Fit } \\
\hline ID & $g_{\text {eff }}$ & $A$ & $\begin{array}{c}\Delta B \\
(\mathrm{mT})\end{array}$ & $\alpha$ & $g_{\text {fit }}$ & $\begin{array}{r}B_{a n} \\
(\mathrm{mT})\end{array}$ & $K_{2} / K_{l}$ & $\begin{array}{c}\sigma \\
(\mathrm{mT})\end{array}$ \\
\hline $\mathrm{A} 1 \mathrm{a}$ & 2.47 & 0.64 & 215 & 0.32 & 2.76 & 149 & -0.04 & 64 \\
$\mathrm{~A} 1 \mathrm{~b}$ & 2.46 & 0.64 & 201 & 0.31 & 2.75 & 144 & -0.04 & 57 \\
$\mathrm{~A} 2 \mathrm{a}$ & 2.53 & 0.69 & 211 & 0.32 & 2.79 & 134 & 0.02 & 59 \\
$\mathrm{~A} 2 \mathrm{~b}$ & 2.48 & 0.68 & 206 & 0.32 & 2.73 & 138 & -0.02 & 59 \\
$\mathrm{~A} 3 \mathrm{a}$ & 2.59 & 0.97 & 266 & 0.43 & 2.63 & 97 & 0.46 & 75 \\
$\mathrm{~A} 3 \mathrm{c}$ & 2.45 & 0.83 & 214 & 0.35 & 2.59 & 134 & -0.08 & 71 \\
$\mathrm{~T} 1 \mathrm{c}$ & 2.16 & 0.77 & 297 & 0.42 & 2.33 & 144 & 0.22 & 71 \\
$\mathrm{~T} 2 \mathrm{~b}$ & 2.26 & 0.80 & 299 & 0.43 & 2.42 & 139 & 0.25 & 72 \\
$\mathrm{~T} 3 \mathrm{c}$ & 2.20 & 0.71 & 228 & 0.34 & 2.40 & 147 & 0.01 & 63 \\
\hline
\end{tabular}


Table 3

Summary of FMR and magnetic parameters of synthetic magnetite samples

\begin{tabular}{|c|c|c|c|c|c|c|c|c|c|c|}
\hline \multicolumn{2}{|c|}{ Sample } & \multicolumn{4}{|c|}{ FMR Spectrum } & \multicolumn{4}{|c|}{ FMR Uniaxial Fit } & \multirow[b]{2}{*}{$\begin{array}{c}k_{\mathrm{ARM}} / \\
\mathrm{IRM} \\
(\mathrm{mm} / \mathrm{A})\end{array}$} \\
\hline ID & $\begin{array}{c}\text { Conc. } \\
\text { (ppm)/ } \\
\text { Pwd Time } \\
\text { (min) }\end{array}$ & $g_{\text {eff }}$ & $A$ & $\begin{array}{c}\Delta B \\
(\mathrm{mT})\end{array}$ & $\alpha$ & $g_{\text {fit }}$ & $\begin{array}{c}B_{\text {an }} \\
(\mathrm{mT})\end{array}$ & $\overline{K_{2} / K_{1}}$ & $\begin{array}{c}\sigma \\
(\mathrm{mT})\end{array}$ & \\
\hline T1a & $60,000 / 0$ & 2.37 & 1.12 & 339 & 0.52262 & 2.31 & 114 & 0.59 & 78 & 0.11 \\
\hline $\mathrm{T} 1 \mathrm{~b}$ & $60,000 / 1$ & 2.21 & 0.96 & 282 & 0.43956 & 2.26 & 117 & 0.34 & 74 & 0.15 \\
\hline T1c & $60,000 / 4$ & 2.11 & 0.87 & 267 & 0.40956 & 2.19 & 123 & 0.25 & 69 & 0.40 \\
\hline $\mathrm{T} 2 \mathrm{a}$ & $6,000 / 0$ & 2.17 & 0.91 & 274 & 0.42322 & 2.24 & 126 & 0.24 & 73 & 0.18 \\
\hline $\mathrm{T} 2 \mathrm{~b}$ & $6,000 / 1$ & 2.16 & 0.92 & 259 & 0.41022 & 2.23 & 123 & 0.20 & 73 & 0.24 \\
\hline $\mathrm{T} 2 \mathrm{c}$ & $6,000 / 4$ & 2.10 & 0.85 & 228 & 0.36794 & 2.19 & 121 & 0.09 & 69 & 0.64 \\
\hline T3a & $400 / 0$ & 2.09 & 0.83 & 230 & 0.36650 & 2.19 & 131 & 0.06 & 67 & 0.59 \\
\hline $\mathrm{T} 3 \mathrm{~b}$ & $400 / 1$ & 2.09 & 0.83 & 222 & 0.35866 & 2.19 & 132 & 0.03 & 67 & 0.74 \\
\hline $\mathrm{T} 3 \mathrm{c}$ & $400 / 4$ & 2.07 & 0.83 & 201 & 0.33808 & 2.17 & 131 & -0.06 & 64 & 1.23 \\
\hline
\end{tabular}

Table 4

Summary of parameters of environmental samples

\begin{tabular}{|c|c|c|c|c|c|}
\hline \multirow[t]{2}{*}{ Sample } & \multicolumn{4}{|c|}{ FMR Spectrum } & \multirow[b]{2}{*}{$\begin{array}{c}k_{\mathrm{ARM}} / \\
\mathrm{IRM} \\
(\mathrm{mm} / \mathrm{A})\end{array}$} \\
\hline & $g_{\text {eff }}$ & $A$ & $\begin{array}{c}\Delta B \\
(\mathrm{mT})\end{array}$ & $\alpha$ & \\
\hline \multicolumn{6}{|l|}{$\begin{array}{l}\text { Andros Island } \\
\text { C51 }\end{array}$} \\
\hline $6 \mathrm{~cm}$ & 2.03 & 0.72 & 143 & 0.26 & 2.54 \\
\hline $14 \mathrm{~cm}$ & 2.01 & 0.71 & 143 & 0.26 & 2.55 \\
\hline $22 \mathrm{~cm}$ & 2.01 & 0.78 & 124 & 0.25 & 3.08 \\
\hline $31 \mathrm{~cm}$ & 2.01 & 0.74 & 124 & 0.25 & 2.49 \\
\hline $42 \mathrm{~cm}$ & 2.02 & 0.80 & 118 & 0.25 & 1.21 \\
\hline $52 \mathrm{~cm}$ & 2.02 & 0.80 & 114 & 0.25 & 0.96 \\
\hline Andros Island & & & & & \\
\hline Mean & 2.04 & 0.77 & 140 & 0.27 & 1.97 \\
\hline Min. & 2.01 & 0.71 & 109 & 0.25 & 0.96 \\
\hline Max. & 2.11 & 0.86 & 192 & 0.32 & 3.08 \\
\hline$D S D P 73-522$ & & & & & \\
\hline Mean & 2.08 & 0.83 & 146 & 0.28 & 1.31 \\
\hline Min. & 2.03 & 0.58 & 136 & 0.26 & 1.07 \\
\hline Max. & 2.17 & 1.01 & 169 & 0.31 & 1.62 \\
\hline Wilson Creek & & & & & \\
\hline Mean & 2.88 & 1.11 & 242 & 0.43 & 0.25 \\
\hline Min. & 2.58 & 0.96 & 207 & 0.40 & 0.25 \\
\hline Max. & 3.34 & 1.33 & 287 & 0.47 & 0.38 \\
\hline
\end{tabular}




\section{Figure Captions}

Figure 1: Synthetic FMR spectra. Generated with $g=2.12$, and (a) no magnetic anisotropy, (b) cubic $B_{a n}=-55 \mathrm{mT}$, as expected for non-interacting cubic magnetite, (c) uniaxial $B_{a n}=100 \mathrm{mT}$, (d) uniaxial $B_{a n}=-100 \mathrm{mT}$, and (e) uniaxial $B_{a n}=100 \mathrm{mT}$. For (a-d), $\sigma=25 \mathrm{mT}$; for $(\mathrm{e}), \sigma=50$ mT. Thick lines show derivative spectra and thin lines show integrated absorption spectra with Gaussian broadening lowered to $\sigma=3 \mathrm{mT}$. Sharp spectra with positive uniaxial anisotropy, as in (c), have two local maxima on the low field side, while sharp spectra with negative uniaxial anisotropy, as in (d), have two local minima on the high field side; these features can be obscured by spectral broadening, as seen in comparison of (c) and (e).

Figure 2: Definitions of basic FMR parameters. Illustrated on a synthetic FMR spectrum of noninteracting, equidimensional magnetite $\left(g=2.12, \sigma=30 \mathrm{mT}\right.$, cubic $\left.B_{a n}=-55 \mathrm{mT}, K_{2} / K_{1}=0.21\right)$. The dark line shows the derivative spectrum and the light line shows the integrated spectrum.

Figure 3: Measurements of intact and altered AMB-1. (a) ARM acquisition curves, (b) ferromagnetic resonance spectra, and (c) low-temperature cycling curves of cultures of AMB-1. In (a), the lower dashed line is a chiton tooth standard for highly interacting magnetite. In (b), thick lines represent room-temperature measurements, thin lines represent $77 \mathrm{~K}$ measurements (where performed), and dashed lines indicate spectral fits.

Figure 4: Measurements of synthetic magnetite. (a) ARM acquisition curves and (b) ferromagnetic resonance spectra of dilutions of synthetic magnetite powder TMB-100. In (a), the upper dashed line is intact AMB-1. The chiton tooth standard shown in Fig. 2a closely follows the line for sample T1a. In (b), thick lines represent room-temperature measurements, thin lines represent $77 \mathrm{~K}$ measurements, and dashed lines indicate spectral fits.

Figure 5: Semi-log plots of the FMR parameters of the synthetic magnetite and AMB-1 samples against $k_{A R M} / I R M$.

Figure 6: Transmission electron micrographs of $\mathrm{Pt}-\mathrm{C}$ replica of synthetic magnetite powder TMB-100 diluted at 6,000 ppm in sucrose. The bright material is the Pt-C replica of the sucrose dilutant, while the dark crystals are magnetite. (a) shows the association between clumps and strings of magnetite particles and the surface of sucrose crystals. (b), (c), and (d) show higher resolution images of the clumps and strings.

Figure 7: Summary of FMR parameters. Plots of (a) effective $g$-factor vs. asymmetry ratio $A$, in the manner of Weiss et al. [20], and (b) full width at half maximum ( $\left.\triangle B_{F W H M}\right)$ vs. $A$. In (a), the dashed lines demarcate the area defined by Weiss et al. [20] as being the domain of magnetosome chains, with $A<1$ and $g_{\text {eff }}<2.12$. In (b), the dashed lines are contours of constant $\alpha$ values, where $\alpha$ is defined in the text. Red and green arrows highlight the dilution trends for, respectively, SDStreated AMB-1 and TMB-100. Parameters for Geobacter metallireducens GS-15, which precipitates extracellular superparamagnetic magnetite, and for the magnetotactic bacteria strains MV-1, MS-1, and MC-1 (arranged in order of increasing $A$ ) are derived from the data of [20].

Figure 8: Measurements through Andros Island core C51. (a) Smoothed ferromagnetic resonance spectra, (b) ARM acquisition curves, and (c) plots of FMR parameters against $k_{A R M} / I R M$. In (a), 
the small peak at $\sim 160 \mathrm{mT}$ is the paramagnetic resonance of $\mathrm{Fe}^{+3}$ and the jaggedness at $\sim 330 \mathrm{mT}$ is the remanent of the $\mathrm{Mn}^{+2}$ resonance, most of which was removed by smoothing. On (b), the upper dashed line is the ARM acquisition curve of intact AMB-1, while the lower dashed line is that of the highly interacting chiton tooth standard. The $31 \mathrm{~cm}$ ARM curve, not shown, nearly follows the $14 \mathrm{~cm}$ curve.

Figure 9: Representative FMR spectra of se (a) Oligocene-Miocene deep-sea muds from DSDP Leg 73 Site 522, in which magnetofossils are a major remanence carrier and (b) silts from the Pleistocene Wilson Creek Formation of Mono Basin, which are magnetically dominated by detrital magnetite. In (a), the dashed line shows by way of comparison AMB-1 sample A3c, which was treated with SDS and diluted in sucrose by powdering for five minutes.

Figure 10: The factor $\alpha$ for model spectra. $\alpha$ is plotted for synthetic spectra with first-order uniaxial anisotropy specified by $B_{a n}$. Contour lines show values of $\sigma$ ranging from $20 \mathrm{mT}$ to 120 $\mathrm{mT}$ in $10 \mathrm{mT}$ steps. In the region occupied by measured magneotactic bacteria and magnetofossil-bearing samples $\left(B_{a n} \geq 75 \mathrm{mT}, \alpha<0.30\right), \alpha$ varies nearly linearly with $\sigma$. 


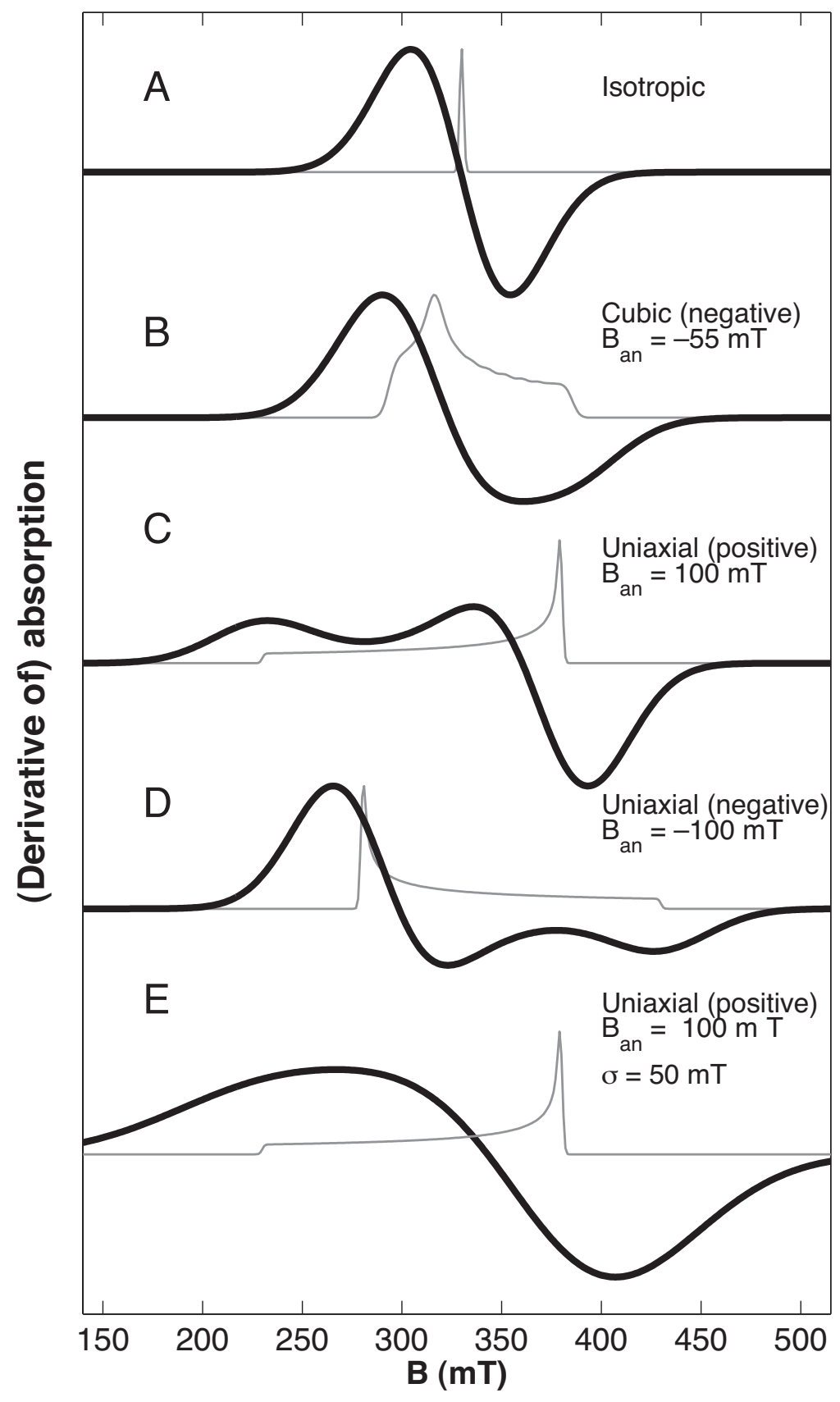

Kopp et al., fig 1 

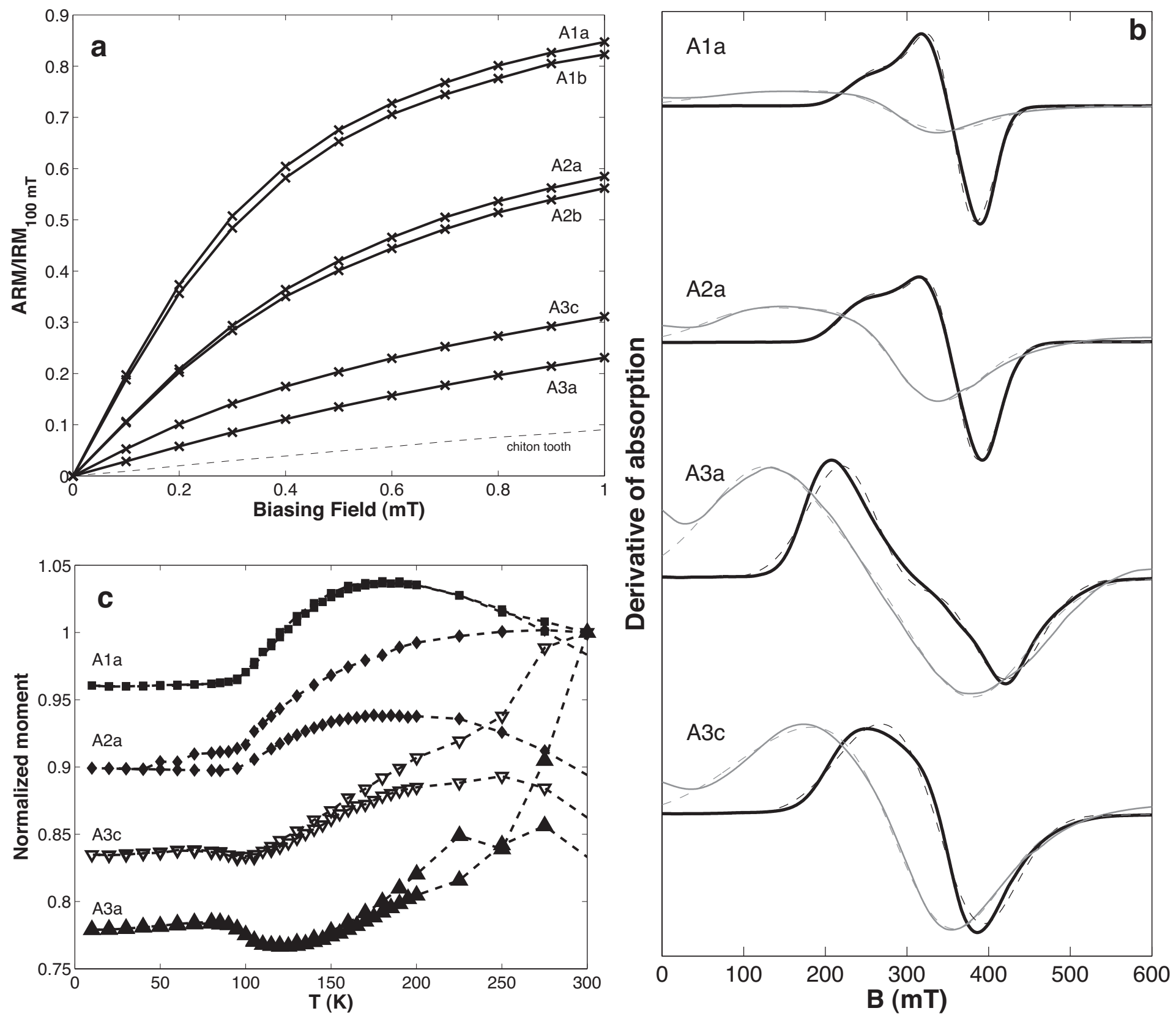

Kopp et al., fig 3 

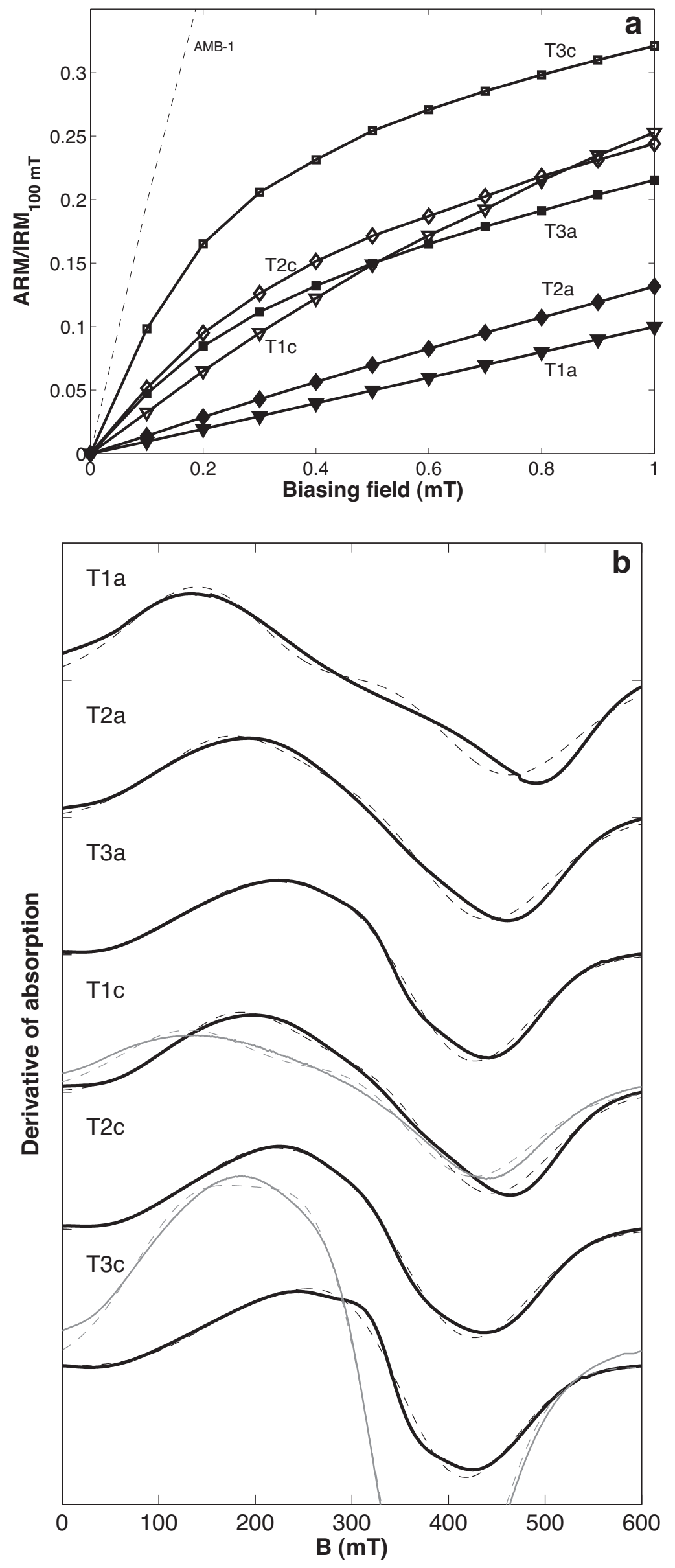

Kopp et al., fig 4 

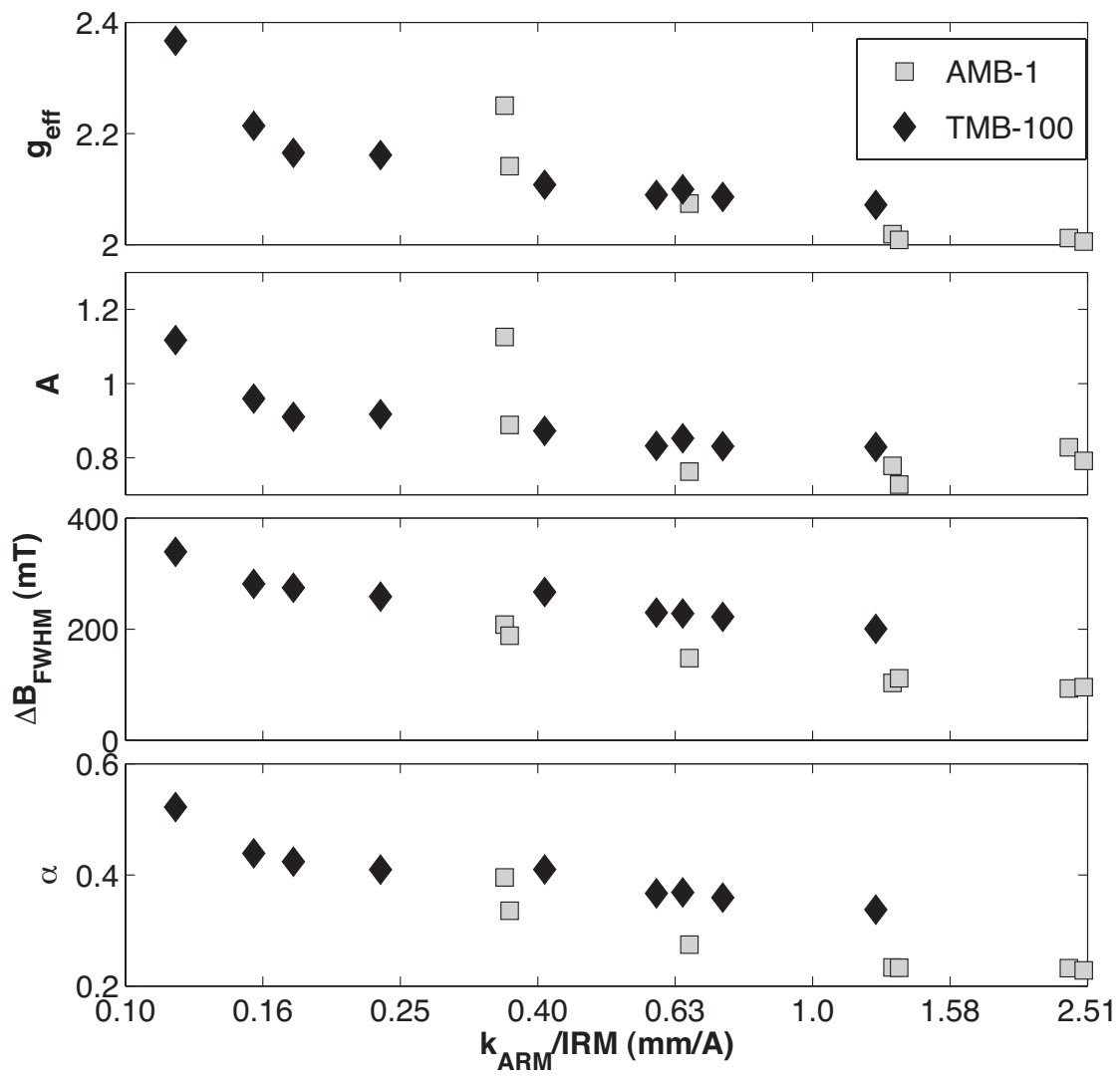

Kopp et al., fig 5 

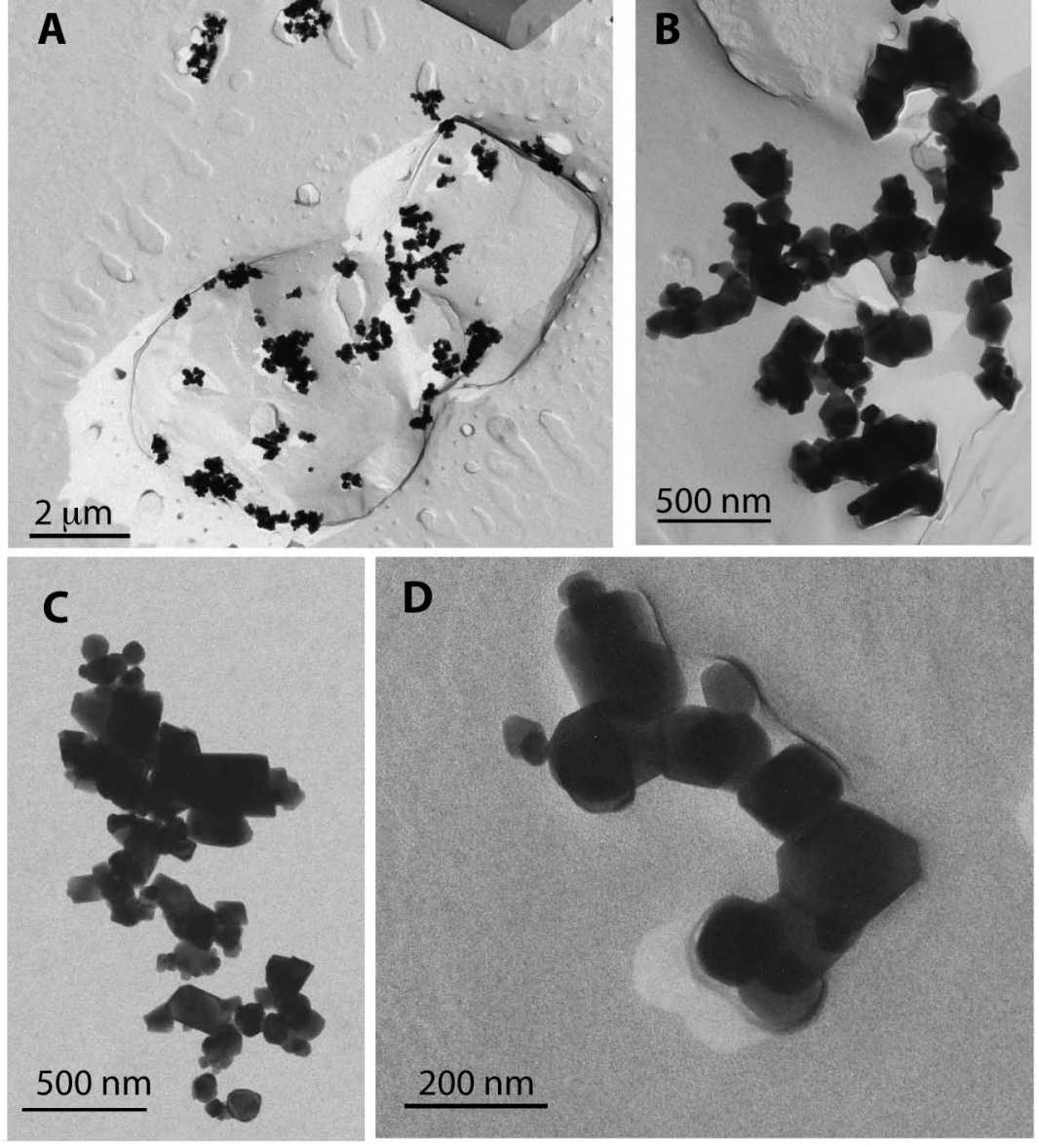

Kopp et al., fig 6 

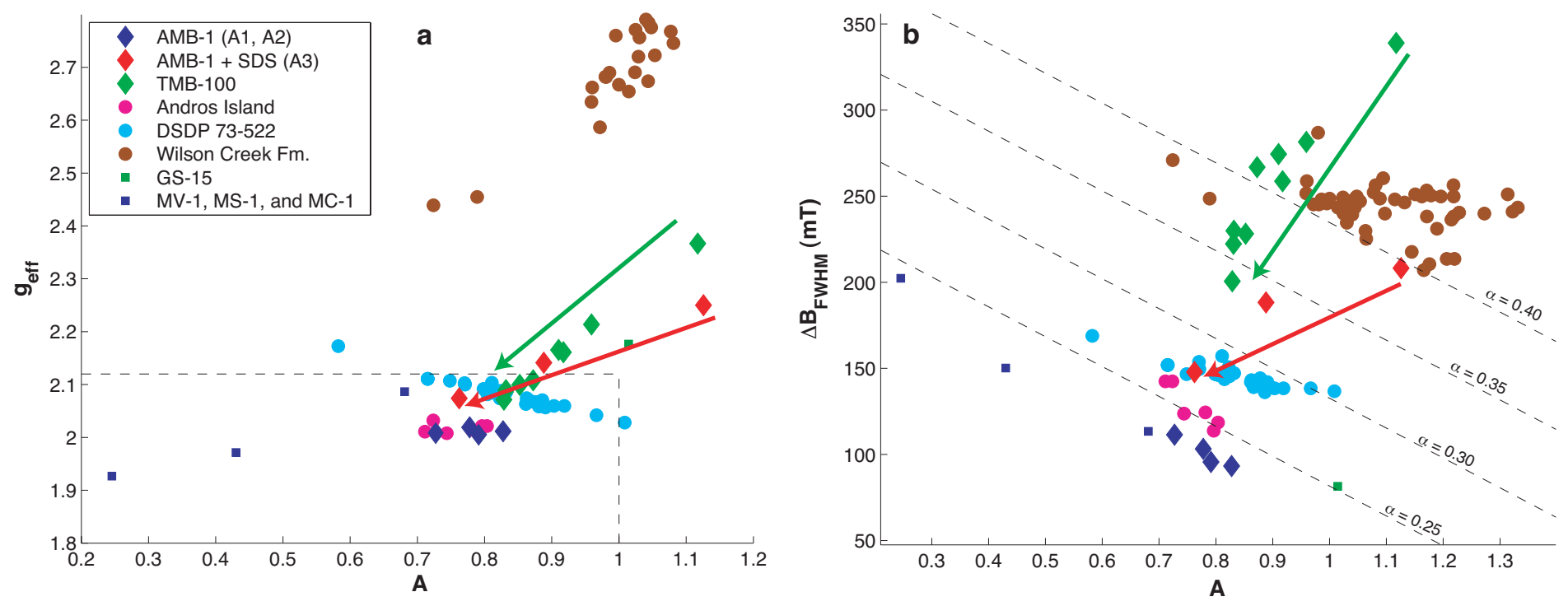

Kopp et al., Fig 7 

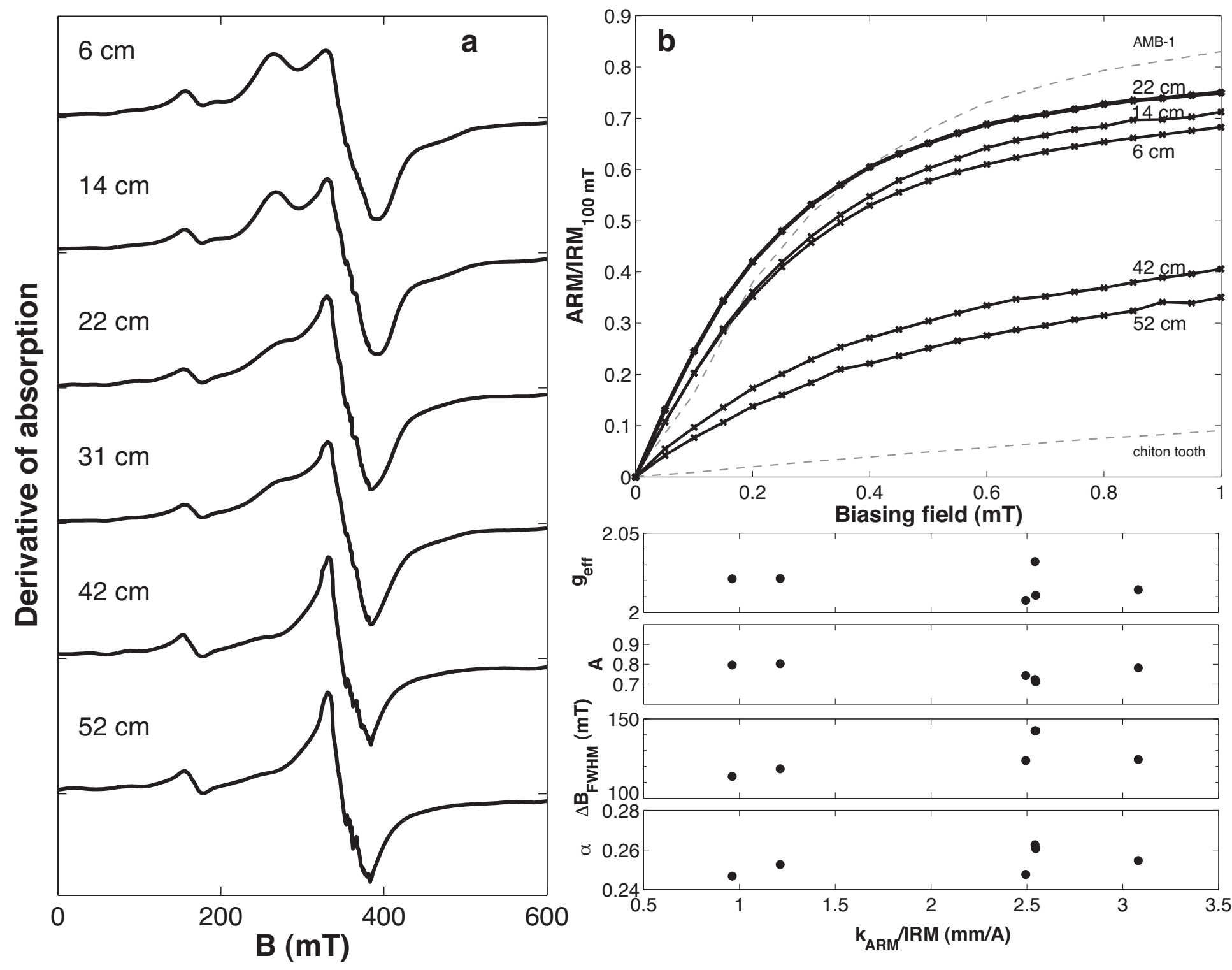

Kopp et al., fig 8 


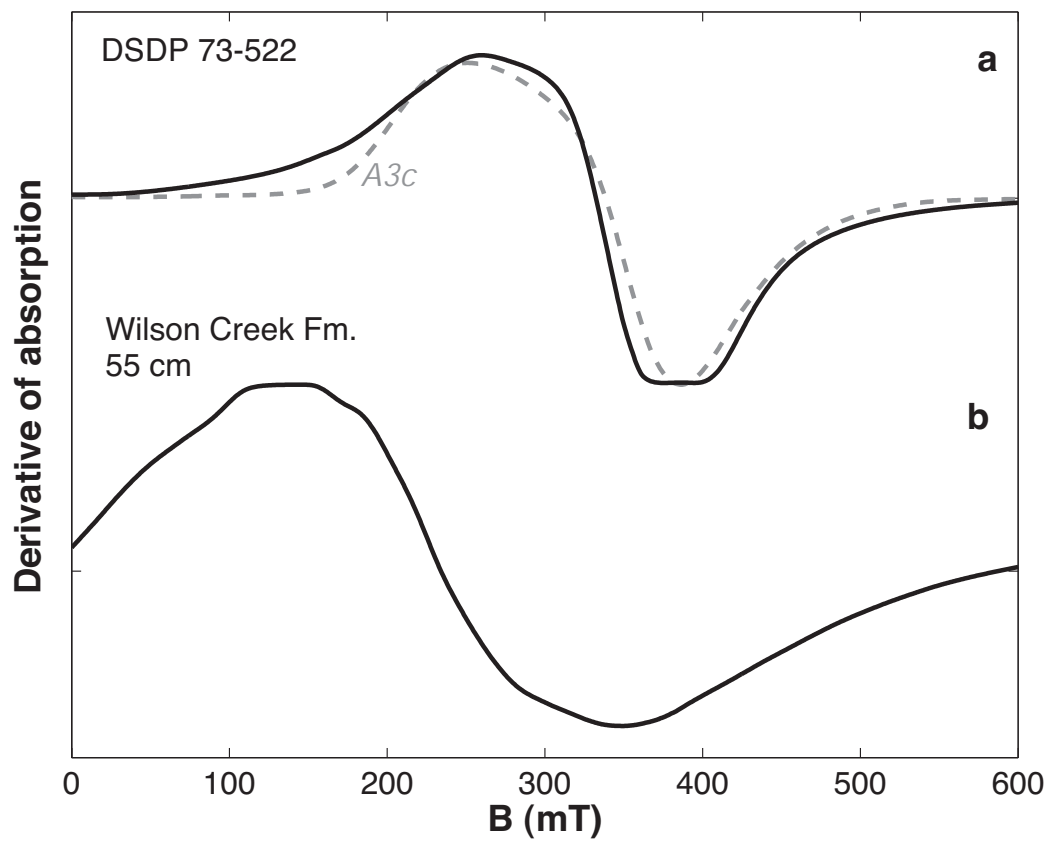

Kopp et al., fig 9 


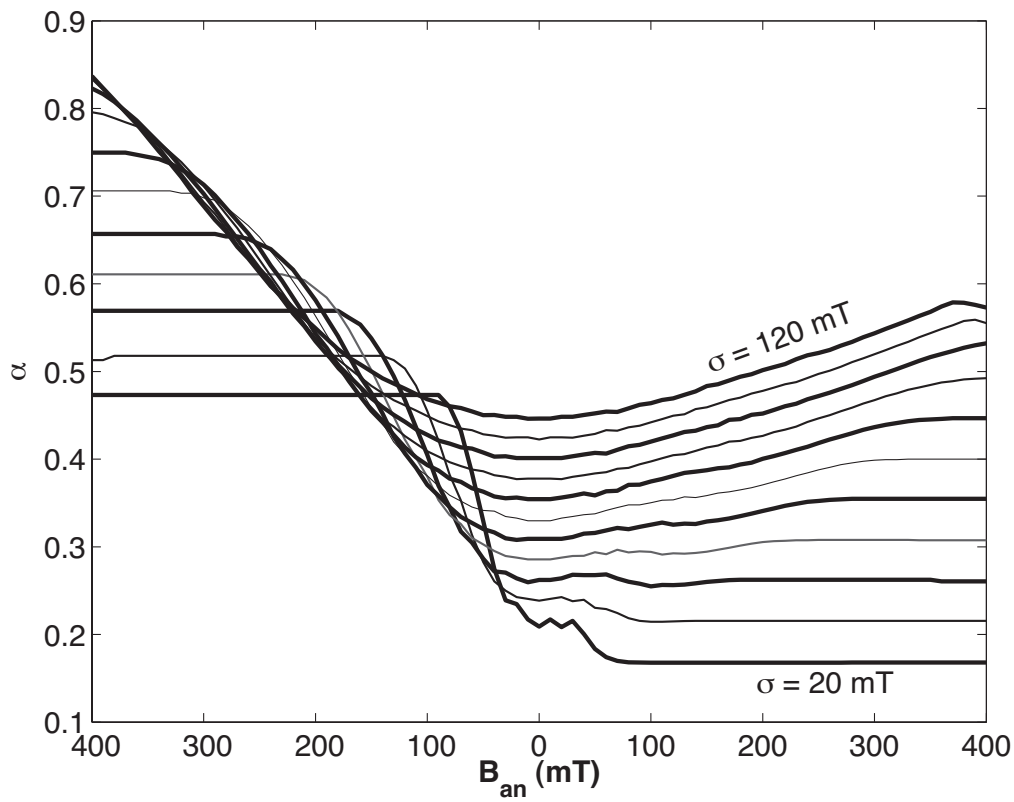

Kopp et al., fig 10 